\title{
The path integral representation kernel of evolution operator in Merton-Garman model
}

\author{
L.F. Blazhyevskyi[1], V.S. Yanishevsky \\ 1 Department for Theoretical Physics, Ivan Franko National University of Lviv, 12 Drahomanov Str., \\ 79005 Lviv, Ukraine \\ 2 Department for Economical Cybernetics and Innovations, Drohobych Ivan Franko State Pedagogical \\ University, 46 Lesja Ukrajinka Str., 82100 Drohobych, Ukraine
}

Received September 7, 2010, in final form February 23, 2011

\begin{abstract}
In the framework of path integral the evolution operator kernel for the Merton-Garman Hamiltonian is constructed. Based on this kernel option formula is obtained, which generalizes the well-known Black-Scholes result. Possible approximation numerical schemes for path integral calculations are proposed.
\end{abstract}

Key words: path integration, evolution operator kernel, option pricing, Black-Scholes formula, Merton-Garman model

PACS: 03.65.-w., 89.65.Gh, 89.65.s, 02.50.r, 02.50.Cw

\section{Introduction}

The path integral method was proposed by R. Feynman as a new approach [1] to the solution of quantum mechanical problems. Nowadays it became one of the most powerful methods in theoretical physics. Many applications (see [2 [5]) of this method are devoted to diverse important problems. In articles [6, 7] describing physical analogies, the path integral method was applied to a series of financial tasks. One of these tasks is the problem of evaluating the option price, initiated in the seminal work [8] which is known as the Black-Scholes model (see appendix [A]). Some generalization of this model is the Merton-Garman model, where there was suggested [6, 7, 9] a suitable dynamics equation of the option price. Just like the Schrodinger equation, the MertonGarman equation is of evolution type. Hence, the path integral method is well fit for presenting the corresponding solution in a closed form and reduces the problem to quadratures. In articles [6, 7] the path integral representation was constructed for the operator evolution kernel (propagator) of a Merton-Garman Hamiltonian.

Meanwhile, while constructing the evolution operator kernel in [6, 7], there were encountered a few shortcomings. In particular, the operator structure of the Merton-Garman Hamiltonian was not taken into account which holds the terms with mutually non-commuting summands by analogy with momentum and coordinate operators of quantum mechanics. In the case under regard, the evolution operator kernel for the Merton-Garman equation is not determined within the Feynman path integral (on the phase space) from the classical Hamiltonian function entailing from the Hamiltonian operator by replacing the momentum and coordinate operators by their classical expressions $\hat{p}_{y} \rightarrow p_{y} \quad\left(\hat{p}_{y}=-\mathrm{i} \partial / \partial y\right)$. Correspondingly, the same holds for the path integral in the configuration space, and thus the classical Lagrange function does not make it possible to correctly determine the evolution operator kernel. The situation mentioned above is well known [2, 3 , $10-12]$ in quantum mechanics and concerns the operator ordering problem. It is known [10] that in the case of terms with non-commuting factors within the Hamiltonian the corresponding action in the path integral still contains additional components. Subject to the Trotter formula approach (which was used in [6, 7]]), the above mentioned components were chosen from the correct form of the resulting Hamiltonian evolution equation based on the obtained evolution operator kernel. As it follows from [10] (see also 11]), since these additional components were not taken into account the 
evolution operator kernel does not correspond to the initial one but rather to some other operator symmetrized over the momentum operators with the Hamiltonian operator. Thus, the evolution operator kernel constructed in [6, 7], does not correspond to the initial Merton-Garman Hamiltonian but to some other Hamiltonian. The determination of these additional components within the Trotter approach is a rather cumbersome procedure [10]. In our work, we use an alternative approach for construction of the path integral evolution operator kernel representation based on the Gaussian path integral within the $T$-product approach [11, 13] (see appendix [B). It is well known that this approach is effective for Hamiltonians with quadratic momentum dependence, that is the case for the Merton-Garman Hamiltonian. We constructed the corresponding path integral evolution kernel representation for the Merton-Garman equation having interpreted the integration variable as the velocity [2, 11]. Moreover, we determined the additional components within the natural Gauss integration procedure assuming the proper symmetrization. Based on this evolution operator kernel we presented an analog of the Black-Scholes formula for a European call option price.

\section{The basic Merton-Garman model setting}

Within the Black-Scholes model [6, 8] two main financial instruments are considered: bank account and stocks. A bank account change $\Pi(t)>0-$ on risk-free instruments and governed by the equation:

$$
\mathrm{d} \Pi(t)=r \Pi(t) \mathrm{d} t
$$

where the value of $r \geqslant 0$ - interest rate. A share price change $S(t) \geqslant 0$ - can be determined from the stochastic equation:

$$
\mathrm{d} S(t)=\phi S(t) \mathrm{d} t+\sigma S(t) R(t) \mathrm{d} t
$$

$\phi \geqslant 0$ - is the expected return on the security $S(t)$ and $\sigma \geqslant 0$ - stock price volatility (the standard deviation). The quantities $\phi, \sigma$ are taken to be constant. The second summand (2) contains a Gaussian random function $R(t)$ (the white noise), whose first moments are equal:

$$
\langle R(t)\rangle=0, \quad\left\langle R(t) R\left(t^{\prime}\right)\right\rangle=\delta\left(t-t^{\prime}\right) .
$$

The brackets above $\langle\ldots\rangle$ denote the Gauss average of the random variable $R(t)$. Stochastic equation (2) is called a geometric (as well as economical) Brown motion [14]. As concerns the classical Langevin equation for the Brown particle velocity [15, 16], a random component of equation (2) is proportional to $S(t)$. The quantity $\mathrm{d} W(t)=R(t) \mathrm{d} t$ in equation (2) is also referred to as the standard Brown motion (or the Wiener process) which describes the independent increases of quantity $R(t)$ with characteristics:

$$
\langle W(t)\rangle=0, \quad\left\langle W(t) W\left(t^{\prime}\right)\right\rangle=\min \left(t, t^{\prime}\right) .
$$

The basic assumptions of the classical option pricing theory are that the option price $C(t, S(t))$ at time $t$ is a continuous function of time and its underlying asset's price $S(t)$. To realize this dependence, it is necessary to impose some relationships. The Black and Scholes idea, concerning the determination of a rational option price [8], was based on a call option payment (premium) $C(t)$ at a moment of time $t(0 \leqslant t \leqslant T)$, considered by an option seller as a minimal capital (investment portfolio), whose investments will permit a contract to get fulfilled. Making use of this aspect, Black and Scholes have determined [6, 8] the following investment portfolio:

$$
C(t, S(t))=\Pi(t)+\frac{\partial C(t, S(t))}{\partial S(t)} S(t) .
$$

The first summand in (4) means the premium part which is placed in the bank account, the second part is assigned for buying shares at market prices $S(t)$. Within the economical sense, namely 
coefficient $\partial C(t, S(t)) / \partial S(t)>0$ determines the amount of shares bought at the price $S(t)$. For option price dynamics equation to be obtained we need to find the change in time of both parts of equality (4), having taken into account equations (1), (2). In particular, in the left hand side of (4) one obtains (for brevity, the $t$-argument is omitted):

$$
\frac{\mathrm{d} C}{\mathrm{~d} t}=\frac{\partial C}{\partial t}+\frac{\partial C}{\partial S} \frac{\mathrm{d} S}{\mathrm{~d} t}+\frac{1}{2} \frac{\partial^{2} C}{\partial S^{2}} \frac{(\mathrm{d} S)^{2}}{\mathrm{~d} t} .
$$

The last summand in (5]) needs to take [6, 14] the relationship $(\mathrm{d} S)^{2}=\sigma^{2} S^{2} \mathrm{~d} t$ into account. Taking now the derivative from the right hand side of (4) and equating it to (5), upon some simplifications, one obtains [6, 8, 14] the Black-Scholes equation:

$$
\frac{\partial C}{\partial t}+r S \frac{\partial C}{\partial S}+\frac{1}{2} \sigma^{2} S^{2} \frac{\partial^{2} C}{\partial S^{2}}=r C
$$

Making change of variables $S=\mathrm{e}^{x}(x \in \mathbb{R})$ we obtain equation (43). Its solution (appendix A) permits to obtain the Black-Scholes formula for the rational option price (55).

As it was noticed above, the volatility $\sigma^{2}$ within the Black-Scholes model is a constant value. Thus, some generalizations of the Black-Scholes model were proposed in which the volatility changes with time. In particular, in a rather general form, the volatility dynamics equation was written [6, 9] as follows:

$$
\mathrm{d} V(t)=\left[\lambda+\mu V(t)+\xi V^{\alpha}(t) R_{2}(t)\right] \mathrm{d} t,
$$

where $V(t)=\sigma^{2}(t), R_{2}(t)$ - Gauss white noise. There are also conditions imposed on the constants $\lambda, \mu, \xi$ which provide the positivity $V(t)>0$. Then, the dynamics share price can be written as follows:

$$
\mathrm{d} S(t)=\phi S(t) \mathrm{d} t+\sqrt{V(t)} S(t) R_{1}(t) \mathrm{d} t .
$$

The correlation Gauss white noise $R_{1}(t), R_{2}(t)$ moments are determined by relationships (3)), as well as by:

$$
\left\langle R_{1}(t) R_{2}\left(t^{\prime}\right)\right\rangle=\rho \delta\left(t-t^{\prime}\right)
$$

with the correlation parameter $-1 \leqslant \rho \leqslant 1$

While deriving the dynamics option price equation the Black-Scholes portfolio property (4) was taken into account. It is easy to see that the relationship structure of (4) gives rise to the summand contraction of $\sim \mathrm{d} S(t) / \mathrm{d} t$, and the equation (6) does not contain the terms proportional to the random component $\sim R(t)$. Meanwhile, the price $S(t)$ is determined at time $t$ (similar to the Brown particle velocity, whose velocity is known at initial moment $t$, but its change is a random variable). Making use of this property while forming the investment portfolio, the following equation was obtained (its more detailed analysis can be found in $[6,7])$ for the option price dynamics:

$$
\frac{\partial C}{\partial t}+r S \frac{\partial C}{\partial S}+(\lambda+\mu V) \frac{\partial C}{\partial V}+\frac{1}{2} V S^{2} \frac{\partial^{2} C}{\partial S^{2}}+\rho \xi V^{1 / 2+\alpha} S \frac{\partial^{2} C}{\partial S \partial V}+\xi^{2} V^{2 \alpha} \frac{\partial^{2} C}{\partial V^{2}}=r C .
$$

Introducing new variables $S=\mathrm{e}^{x}, V=\mathrm{e}^{y}(x, y \in \mathbb{R})$ equation (10) can be written down as follows:

$$
\frac{\partial C(t)}{\partial t}=H_{\mathrm{MG}} C(t)
$$

where we denoted the quantity:

$$
\begin{aligned}
H_{\mathrm{MG}}= & -\frac{\mathrm{e}^{y}}{2} \frac{\partial^{2}}{\partial x^{2}}+\left(\frac{\mathrm{e}^{y}}{2}-r\right) \frac{\partial}{\partial x}+r-\left(\lambda \mathrm{e}^{-y}+\mu-\frac{\xi^{2}}{2} \mathrm{e}^{2 y(\alpha-1)}\right) \frac{\partial}{\partial y}- \\
& -\rho \xi \mathrm{e}^{y(\alpha-1 / 2)} \frac{\partial^{2}}{\partial x \partial y}-\frac{\xi^{2}}{2} \mathrm{e}^{2 y(\alpha-1)} \frac{\partial^{2}}{\partial y^{2}},
\end{aligned}
$$

which means exactly the Merton-Garman Hamiltonian. It is easy to see that the first three summands in (12) correspond to the Black-Scholes Hamiltonian term, while the next ones are owing to the stochastic dynamics of the volatility. 


\section{The path integral representation of the Merton-Garman equation}

The continuous solutions to equation (46), which is understood in the standard generalized sense, can be represented in the form:

$$
C(t)=\mathrm{e}^{-\tau H_{\mathrm{MG}}} C(T),
$$

where $C(T)$ is the value of the option price at time $t$, which is determined by function (53). The evolution operator kernel in (13), similar to (48), can be determined by the relationship:

$$
g\left(\tau, x-x_{0}, y, y_{0}\right)=\mathrm{e}^{-\tau H_{\mathrm{MG}}} \delta\left(x-x_{0}\right) \delta\left(y-y_{0}\right)
$$

Since the Hamiltonian coefficients in (12) are independent of the variable $x$, based on the experience at the construction of the Black-Scholes Hamiltonian and its evolution operator kernel, one can make the Fourier transformation with respect to the $x$-variable and as a result, one obtains the Hamiltonian (12) in the form:

$$
\tilde{H}_{\mathrm{MG}}(k)=h_{0}(k, y)+h_{1}(k, y) \frac{\partial}{\partial y}-\frac{\xi^{2}}{2}\left(\mathrm{e}^{y(\alpha-1)} \frac{\partial}{\partial y}\right)^{2},
$$

where we have denoted:

$$
\begin{gathered}
h_{0}(k, y)=\frac{\mathrm{e}^{y}}{2} k^{2}+\mathrm{i}\left(\frac{\mathrm{e}^{y}}{2}-r\right) k+r, \\
h_{1}(k, y)=-\mathrm{i} \rho \xi \mathrm{e}^{y(\alpha-1 / 2)} k-\left(\lambda \mathrm{e}^{-y}+\mu-\frac{\xi^{2} \alpha}{2} \mathrm{e}^{2 y(\alpha-1)}\right) .
\end{gathered}
$$

Respectively, the Fourier image of the kernel (14) is determined as:

$$
\tilde{g}\left(\tau, k, y, y_{0}\right)=\mathrm{e}^{-\tau \tilde{H}_{\mathrm{MG}}(k)} \delta\left(y-y_{0}\right)
$$

\subsection{The case $\alpha=1$}

Consider the simplest case $\alpha=1$ where the coefficient at the operator $\partial^{2} / \partial y^{2}$ does not depend on the variable $y$. Having chosen the complete square form in the Hamiltonian (15), one obtains:

$$
\tilde{H}_{\mathrm{MG}}(k)=-\frac{1}{2}\left(\xi \frac{\partial}{\partial y}-\frac{h(k, y)}{\xi}\right)^{2}+h_{0}(k, y)+\frac{1}{2} \frac{h^{2}(k, y)}{\xi^{2}}-\frac{1}{2} h^{\prime}(k, y) .
$$

Here the quantity $h(k, y)$ equals the value $h_{1}(k, y)$ (16) for $\alpha=1$ :

$$
h(k, y)=-\mathrm{i} \rho \xi \mathrm{e}^{y / 2} k-\left(\lambda \mathrm{e}^{-y}+\mu-\xi^{2} / 2\right)
$$

and the summand $h^{\prime}(k, y)$ means the derivative of the $h(k, y)$ with respect to the variable $y$ :

$$
h^{\prime}(k, y)=\lambda \mathrm{e}^{-y}-\mathrm{i} k \frac{1}{2} \rho \xi \mathrm{e}^{y / 2}
$$

Namely, a similar summand, as we will show below, was not taken into account in articles [ㅈ, 7]. In our case, this appears to have been caused by the corresponding symmetrization performed in (19). One can also observe that this summand is important for the Hamiltonian (19) (in the coordinate representation $k \rightarrow-\mathrm{i} \partial / \partial x)$ whose form would take an incomplete form:

$$
\begin{aligned}
H_{\mathrm{MG}}^{*}= & -\frac{\mathrm{e}^{y}}{2} \frac{\partial^{2}}{\partial x^{2}}+\left(\frac{\mathrm{e}^{y}}{2}-r\right) \frac{\partial}{\partial x}+r-\left(\mu-\frac{\xi^{2}}{2}\right) \frac{\partial}{\partial y}-\frac{\lambda}{2}\left(\mathrm{e}^{-y} \frac{\partial}{\partial y}+\frac{\partial}{\partial y} \mathrm{e}^{-y}\right)- \\
& -\frac{\rho \xi}{2} \frac{\partial}{\partial x}\left(\mathrm{e}^{y / 2} \frac{\partial}{\partial y}+\frac{\partial}{\partial y} \mathrm{e}^{y / 2}\right)-\frac{\xi^{2}}{2} \frac{\partial^{2}}{\partial y^{2}} \cdot
\end{aligned}
$$


It is seen that that summands with non-commuting factors in (20) are written in a symmetrized form. Namely, as it was already mentioned above, the path integral representation of the evolution operator kernel, constructed in [6, 7], corresponds to (20) in contrast to the initial Hamiltonian (12) (for $\alpha=1$ ).

The evolution operator in (18) can be transformed by using the expression (56):

$$
\begin{aligned}
& \mathrm{e}^{-\tau \tilde{H}_{\mathrm{MG}}(k)}=\mathcal{N}(\tau) \int \mathcal{D} v \exp \left(-\frac{1}{2} \int_{0}^{\tau} v^{2}(t) \mathrm{d} t\right) \times \\
& \times T \exp \left(\int_{0}^{\tau}\left[-h_{0}(k, y)-\frac{1}{2 \xi^{2}} h^{2}(k, y)+\frac{1}{2} h^{\prime}(k, y)+\frac{1}{\xi} v(t) h(k, y)\right] \mathrm{d} t-\int_{0}^{\tau} \xi v(t) \mathrm{d} t \frac{\partial}{\partial y}\right) .
\end{aligned}
$$

The latter summand in the $T$-exponent contains a shift-operator which can be disentangled with respect to (63), since the $T$-exponent becomes a usual one:

$$
\begin{aligned}
T \exp \left(-\int_{0}^{\tau} \xi v(t) \mathrm{d} t \frac{\partial}{\partial y}\right) & =\exp \left(-\int_{0}^{\tau} \xi v(t) \mathrm{d} t \frac{\partial}{\partial y}\right), \\
{\left[T \exp \left(-\int_{0}^{\tau} \xi v(t) \mathrm{d} t \frac{\partial}{\partial y}\right)\right]^{-1} } & =\exp \left(\int_{0}^{\tau} \xi v(t) \mathrm{d} t \frac{\partial}{\partial y}\right) .
\end{aligned}
$$

Respectively, for the operator kernel (18), upon simple transformations which are omitted, one can obtain:

$$
\begin{aligned}
\tilde{g}\left(\tau, k, y, y_{0}\right)= & \overline{\mathcal{N}}(\tau) \int \mathcal{D} v \exp \left(-\frac{1}{2 \xi^{2}} \int_{0}^{\tau}[v(t)-h(k, \tilde{y})]^{2} \mathrm{~d} t-\int_{0}^{\tau} h_{0}(k, \tilde{y}) \mathrm{d} t\right) \times \\
& \times \exp \left(\frac{1}{2} \int_{0}^{\tau} h^{\prime}(k, \tilde{y}) \mathrm{d} t\right) \delta\left(y-y_{0}-\int_{0}^{\tau} v(t) \mathrm{d} t\right),
\end{aligned}
$$

where we denoted $\tilde{y}=y-\int_{t}^{\tau} v\left(t^{\prime}\right) \mathrm{d} t^{\prime}$ and the constant $\overline{\mathcal{N}}(\tau)$ is determined from the condition:

$$
\overline{\mathcal{N}}(\tau) \int \mathcal{D} v \exp \left(-\frac{1}{2 \xi^{2}} \int_{0}^{\tau} v^{2}(t) \mathrm{d} t\right)=1
$$

The path integral (22) is defined in the space of velocities [2, 11]. The quantity

$$
L(k)=\frac{1}{2 \xi^{2}}[v-h(k, y)]^{2}+h_{0}(k, y)
$$

means a Lagrangian function (for imaginary time) and the summand $-h^{\prime}(k, y) / 2$ - the additional term. Relationship (22) determines the Fourier image with respect to $x$-variable. For the Fourier inverse transform one needs to separate in (22) the $k$-dependence:

$$
\tilde{g}\left(\tau, k, y, y_{0}\right)=\mathrm{e}^{-\tau r} \overline{\mathcal{N}}(\tau) \int \mathcal{D} v \mathrm{e}^{S_{0}} \exp \left(-\frac{1}{2} \tau \tilde{\sigma}^{2} k^{2}-\mathrm{i} \tau k\left[\tilde{\sigma}^{2} / 2-\tilde{r}\right]\right) \delta\left(y-y_{0}-\int_{0}^{\tau} v(t) \mathrm{d} t\right)
$$


where we denoted:

$$
\begin{aligned}
S_{0} & =-\frac{1}{2 \xi^{2}} \int_{0}^{\tau}\left[v(t)+\lambda \mathrm{e}^{-\tilde{y}}+\mu-\xi^{2} / 2\right]^{2} \mathrm{~d} t+\frac{1}{2} \lambda \int_{0}^{\tau} \mathrm{e}^{-\tilde{y}} \mathrm{~d} t, \\
\tilde{\sigma}^{2} & =\left(1-\rho^{2}\right) \frac{1}{\tau} \int_{0}^{\tau} \mathrm{e}^{\tilde{y}} \mathrm{~d} t, \\
\tilde{r} & =r-\frac{1}{2} \rho^{2} \frac{1}{\tau} \int_{0}^{\tau} \mathrm{e}^{\tilde{y}} \mathrm{~d} t+\rho\left(\frac{\xi}{4}-\frac{\mu}{\xi}\right) \frac{1}{\tau} \int_{0}^{\tau} \mathrm{e}^{\tilde{y} / 2} \mathrm{~d} t-\frac{\rho}{\xi} \frac{1}{\tau} \int_{0}^{\tau} \mathrm{e}^{\tilde{y} / 2} v(t) \mathrm{d} t-\frac{\lambda \rho}{\xi} \frac{1}{\tau} \int_{0}^{\tau} \mathrm{e}^{-\tilde{y} / 2} \mathrm{~d} t .
\end{aligned}
$$

Performing the inverse Fourier transform we obtain the evolution operator kernel:

$$
\begin{aligned}
g\left(\tau, x-x_{0}, y, y_{0}\right) & =\overline{\mathcal{N}}(\tau) \int \mathcal{D} v \mathrm{e}^{S_{0}} \frac{\mathrm{e}^{-\tau r}}{\sqrt{2 \pi \tau \tilde{\sigma}^{2}}} \times \\
& \times \exp \left(-\frac{1}{2} \frac{\left(x-x_{0}+\tau\left[\tilde{r}-\tilde{\sigma}^{2} / 2\right]\right)^{2}}{\tau \tilde{\sigma}^{2}}\right) \delta\left(y-y_{0}-\int_{0}^{\tau} v(t) \mathrm{d} t\right) .
\end{aligned}
$$

Thus, formula (25) is a path integral representation of the evolution operator kernel for the MertonGarman Hamiltonian in the case $\alpha=1$. As it was mentioned earlier, the comparison of this result with the formula from [6, 7] shows that the latter does not contain the second summand in $S_{0}$, as well as there is a change in the corresponding numerical coefficient in the third summand $\tilde{r}$. Recall that in [6, 7] there is used a Feynman path integral defined in the configurational phase space, in contrast to the kernel (25) which is represented in the velocity phase space. The connection between both representations can be found in appendix C

Making use of this kernel, we obtain a generalization of the Black-Scholes result (55) for the option price. For this to be done, we need to multiply the kernel $g\left(\tau, x-x_{0}, y, y_{0}\right)$ by the payment function (53) and integrate over the variables $x_{0}, y_{0}$. Since function (53) does not depend on $y_{0}$, the integral with respect to $y_{0}$ is easily performed owing to the presence of $\delta$-function. As a result we obtain:

$$
C(t, S(t))=\overline{\mathcal{N}}(\tau) \int \mathcal{D} v \mathrm{e}^{S_{0}}\left[S(t) \mathrm{e}^{\tau \Delta r} N\left(\tilde{d}_{+}\right)-\mathrm{e}^{-\tau r} K N\left(\tilde{d}_{-}\right)\right]
$$

where we denoted $\Delta r=\tilde{r}-r$ and quantities $\tilde{d}_{ \pm}$are determined by (55) for $d_{ \pm}$by means of the change $r \rightarrow \tilde{r}, \sigma \rightarrow \tilde{\sigma}$.

\subsection{The case of arbitrary $\alpha$-values}

In this case, in order to obtain the path integral representation of the evolution operator we will act the following way. First, we will make use of the path integral (56) for linearization of the third summand in (15):

$$
\begin{aligned}
\mathrm{e}^{-\tau \tilde{H}_{\mathrm{MG}}(k)} & =\mathcal{N}(\tau) \int \mathcal{D} w \exp \left(-\frac{1}{2} \int_{0}^{\tau} w^{2}(t) \mathrm{d} t\right) \times \\
& \times T \exp \left(-\int_{0}^{\tau} h_{0}(k, y) \mathrm{d} t-\int_{0}^{\tau}\left[h_{1}(k, y)+\xi w(t) \mathrm{e}^{y(\alpha-1)}\right] \frac{\partial}{\partial y} \mathrm{~d} t\right) .
\end{aligned}
$$


Then, we use the path integral (61) for linearization of the summand with the derivation operator (27). Preliminarily, let us denote:

$$
\begin{aligned}
& A(t)=-\frac{1}{2}\left[h_{1}(k, y)+\xi w(t) \mathrm{e}^{y(\alpha-1)}\right], \quad B(t)=\frac{\partial}{\partial y} \\
& {[A(t), B(t)]_{-}=\frac{1}{2}\left[h_{1}^{\prime}(k, y)+(\alpha-1) \xi w(t) \mathrm{e}^{y(\alpha-1)}\right]=-A^{\prime}(t) .}
\end{aligned}
$$

The sense of the previous transformations, as we observed before, is in splitting a shift-operator which permits to perform the $T$-operation. As a result, for the $T$-exponent (27) we obtain the relationship:

$$
\begin{aligned}
& T \exp \left(-\int_{0}^{\tau} h_{0}(k, y) \mathrm{d} t-\int_{0}^{\tau}\left[h_{1}(k, y)+\xi w(t) \mathrm{e}^{y(\alpha-1)}\right] \frac{\partial}{\partial y} \mathrm{~d} t\right)= \\
& =\mathcal{N}(\tau)^{2} \int \mathcal{D} v \mathcal{D} u \exp \left(-\frac{1}{2} \int_{0}^{\tau} v^{2}(t) \mathrm{d} t-\mathrm{i} \int_{0}^{\tau} v(t) u(t) \mathrm{d} t\right) \times \\
& \times T \exp \left(-\int_{0}^{\tau}\left\{[v(t)+2 \mathrm{i} u(t)] A(t)+v(t) \frac{\partial}{\partial y}+A^{\prime}(t)+h_{0}(k, y)\right\} \mathrm{d} t\right) .
\end{aligned}
$$

Disentangling the exponent containing the shift-operator and substituting it into (27), we write down the evolution operator in the form:

$$
\mathrm{e}^{-\tau \tilde{H}_{\mathrm{MG}}(k)}=\mathcal{N}(\tau) \int \mathcal{D} v \exp \left(-\frac{1}{2} \int_{0}^{\tau} v^{2}(t) \mathrm{d} t\right) \Phi(v) \exp \left(-\int_{0}^{\tau} h_{0}(k, \tilde{y}) \mathrm{d} t\right) \exp \left(-\int_{0}^{\tau} v(t) \mathrm{d} t \frac{\partial}{\partial y}\right)
$$

where we denoted:

$$
\begin{aligned}
\Phi(v) & =\mathcal{N}(\tau) \int \mathcal{D} u \exp \left(-\mathrm{i} \int_{0}^{\tau} v(t) u(t) \mathrm{d} t\right) \mathcal{N}(\tau) \int \mathcal{D} w \exp \left(-\frac{1}{2} \int_{0}^{\tau} w^{2}(t) \mathrm{d} t\right) \times \\
& \times \exp \left(-\int_{0}^{\tau}\left\{[v(t)+2 \mathrm{i} u(t)] \tilde{A}(t)+\tilde{A}^{\prime}(t)\right\} \mathrm{d} t\right) .
\end{aligned}
$$

Let us note here that $(\sim)$-barred quantities differ from those, used before, by the change to $y \rightarrow \tilde{y}$, where $\tilde{y}=y-\int_{t}^{\tau} v\left(t^{\prime}\right) \mathrm{d} t^{\prime}$.

While calculating $\Phi(v)$ it is necessary to perform integration with respect to variables $w(t)$ and $u(t)$. Since the mentioned integrals are Gaussian, they can be performed in closed form. Upon simple but a bit cumbersome calculations, which are omitted, we obtain:

$$
\begin{aligned}
& \mathrm{e}^{-\tau \tilde{H}(k)}=\overline{\mathcal{N}}(\tau) \int \mathcal{D} v \prod_{t} \mathrm{e}^{-\tilde{y}(\alpha-1)} \exp \left(-\frac{1}{2 \xi^{2}} \int_{0}^{\tau} \frac{\left[v(t)-h_{1}(k, \tilde{y})\right]^{2}}{\mathrm{e}^{2 \tilde{y}(\alpha-1)}} \mathrm{d} t\right) \times \\
& \quad \times \exp \left(-\int_{0}^{\tau} h_{0}(k, \tilde{y}) \mathrm{d} t+\frac{1}{2} \int_{0}^{\tau} h_{1}^{\prime}(k, \tilde{y}) \mathrm{d} t+\frac{1}{2}(\alpha-1) \int_{0}^{\tau}\left[v(t)-h_{1}(k, \tilde{y})\right] \mathrm{d} t\right) \exp \left(-\int_{0}^{\tau} v(t) \mathrm{d} t \frac{\partial}{\partial y}\right) .
\end{aligned}
$$

Respectively, for the Fourier representation of the kernel (18) one obtains the relationship:

$$
\tilde{g}\left(\tau, k, y, y_{0}\right)=\overline{\mathcal{N}}(\tau) \int \mathcal{D} v \prod_{t} \mathrm{e}^{-\tilde{y}(\alpha-1)} \exp \left(-\int_{0}^{\tau} L(k, v(t), \tilde{y}) \mathrm{d} t\right) \delta\left(y-y_{0}-\int_{0}^{\tau} v(t) \mathrm{d} t\right)
$$


where we denoted the function:

$$
L(k, v, y)=\frac{1}{2 \xi^{2}} \frac{\left[v-h_{1}(k, y)\right]^{2}}{\mathrm{e}^{2 y(\alpha-1)}}+h_{0}(k, y)-\frac{1}{2} h_{1}^{\prime}(k, y)+\frac{1}{2}(\alpha-1)\left[v-h_{1}(k, y)\right] .
$$

Let us mention here that the representation (32) corresponds to the structure [2, 11, 12] of the path integrals subject to the curvilinear coordinates. As is seen from (32) the kernel is not determined by means of a "classical" Lagrange function which is assigned to the Hamiltonian (12). It is not hard to observe that this Lagrange function (for the imaginary time) is determined by the relationship:

$$
L_{0}(k, v, y)=\frac{1}{2 \xi^{2}} \frac{[v-\bar{h}(k, y)]^{2}}{\mathrm{e}^{2 y(\alpha-1)}}+h_{0}(k, y),
$$

where we denoted

$$
\bar{h}(k, y)=-\mathrm{i} \rho \xi \mathrm{e}^{y(\alpha-1 / 2)} k-\left(\lambda \mathrm{e}^{-y}+\mu-\frac{\xi^{2}}{2} \mathrm{e}^{2 y(\alpha-1)}\right),
$$

the quantity $h_{0}(k, y)$ is determined by (16). Note also that $\bar{h}(k, y)$ is different from $h_{1}(k, y)$ (17) by a factor $\alpha$ in the last summand. It is evident that the difference between $L(k, v, y)$ and $L_{0}(k, v, y)$ determines the above mentioned additional terms. The corresponding terms are not taken into account in [6] for the constructed evolution operator kernel of the Hamiltonian (12). Note also that at the change $L(k, v, y) \rightarrow L_{0}(k, v, y)$ in (32), the obtained kernel will correspond not to operator (12), but to its analog $H_{\mathrm{MG}}^{*}$, being in some way symmetrized over operators $\partial / \partial y$. The structure $H_{\mathrm{MG}}^{*}$ can be determined, in particular, making use of the approach devised in [11].

The next step, having split the dependence on the $k$-variable, one can obtain:

$$
\begin{aligned}
\tilde{g}\left(\tau, k, y, y_{0}\right) & =\mathrm{e}^{-\tau r} \overline{\mathcal{N}}(\tau) \int \mathcal{D} v \prod_{t} \mathrm{e}^{-\tilde{y}(\alpha-1)} \mathrm{e}^{S_{0}} \times \\
& \times \exp \left(-\frac{1}{2} \tau \tilde{\sigma}^{2} k^{2}-\mathrm{i} \tau k\left[\tilde{\sigma}^{2} / 2-\tilde{r}\right]\right) \delta\left(y-y_{0}-\int_{0}^{\tau} v(t) \mathrm{d} t\right),
\end{aligned}
$$

where we denoted:

$$
\begin{aligned}
& S_{0}=-\frac{1}{2 \xi^{2}} \int_{0}^{\tau} \frac{[v(t)-h(\tilde{y})]^{2}}{\mathrm{e}^{2 \tilde{y}(\alpha-1)}} \mathrm{d} t+\frac{1}{2} \int_{0}^{\tau} h^{\prime}(\tilde{y}) \mathrm{d} t+\frac{1}{2}(\alpha-1) \int_{0}^{\tau}[v(t)-h(\tilde{y})] \mathrm{d} t \\
& \tilde{\sigma}^{2}=\left(1-\rho^{2}\right) \frac{1}{\tau} \int_{0}^{\tau} \mathrm{e}^{\tilde{y}} \mathrm{~d} t \\
& \tilde{r}=r-\rho^{2} \frac{1}{\tau} \int_{0}^{\tau} \mathrm{e}^{\tilde{y}} \mathrm{~d} t-\frac{\rho \xi}{4} \frac{1}{\tau} \int_{0}^{\tau} \mathrm{e}^{\tilde{y}(\alpha-1 / 2)} \mathrm{d} t-\frac{\rho}{\xi} \frac{1}{\tau} \int_{0}^{\tau}[v(t)-h(\tilde{y})] \mathrm{e}^{\tilde{y}(3 / 2-\alpha)} \mathrm{d} t \\
& h(y)=-\left(\lambda \mathrm{e}^{-y}+\mu-\frac{\xi^{2} \alpha}{2} \mathrm{e}^{2 y(\alpha-1)}\right) .
\end{aligned}
$$

Here $h^{\prime}(y)$ means the derivative of function with respect to a suitable argument. Performing the inverse Fourier transform we obtain the evolution operator kernel in the case of arbitrary $\alpha$-values.

$$
\begin{aligned}
g\left(\tau, x-x_{0}, y, y_{0}\right) & =\overline{\mathcal{N}}(\tau) \int \mathcal{D} v \prod_{t} \mathrm{e}^{-\tilde{y}(\alpha-1)} \mathrm{e}^{S_{0}} \frac{\mathrm{e}^{-\tau r}}{\sqrt{2 \pi \tau \tilde{\sigma}^{2}}} \times \\
& \times \exp \left(-\frac{1}{2} \frac{\left(x-x_{0}+\tau\left[\tilde{r}-\tilde{\sigma}^{2} / 2\right]\right)^{2}}{\tau \tilde{\sigma}^{2}}\right) \delta\left(y-y_{0}-\int_{0}^{\tau} v(t) \mathrm{d} t\right) .
\end{aligned}
$$


Then, the generalization of the Black-Scholes for the option price will take the form:

$$
C(t, S(t))=\overline{\mathcal{N}}(\tau) \int \mathcal{D} v \prod_{t} \mathrm{e}^{-\tilde{y}(\alpha-1)} \mathrm{e}^{S_{0}}\left[S(t) \mathrm{e}^{\tau \Delta r} N\left(\tilde{d}_{+}\right)-\mathrm{e}^{-\tau r} K N\left(\tilde{d}_{-}\right)\right] .
$$

\section{Some comments concerning path integral calculations}

Works [6, 7, 17] present the formulas for the kernel calculation (65) in the case $\alpha=1, \lambda=0$. Meanwhile, the sub-integrand in (65) was represented as a three-tuple series:

$$
\begin{aligned}
& \frac{1}{\sqrt{2 \pi \tau \tilde{\sigma}^{2}}} \exp \left(-\frac{1}{2} \frac{\left(x-x_{0}+\tau\left[\tilde{r}-\tilde{\sigma}^{2} / 2\right]\right)^{2}}{\tau \tilde{\sigma}^{2}}\right) \rightarrow \\
& \rightarrow \sum_{n, m, k} a_{n m k}\left(\frac{1}{\tau} \int_{0}^{\tau} \mathrm{e}^{y(t)} \mathrm{d} t\right)^{n}\left(\frac{1}{\tau} \int_{0}^{\tau} \mathrm{e}^{y(t) / 2} \mathrm{~d} t\right)^{m}\left(\mathrm{e}^{y(0) / 2}\right)^{k} .
\end{aligned}
$$

Then, for a general series term:

$$
\int_{-\infty}^{\infty} \mathrm{d} y_{0} \int_{y_{0}}^{y} \mathcal{D}^{\prime} y \mathrm{e}^{S_{0}}\left(\frac{1}{\tau} \int_{0}^{\tau} \mathrm{e}^{y(t)} \mathrm{d} t\right)^{n}\left(\frac{1}{\tau} \int_{0}^{\tau} \mathrm{e}^{y(t) / 2} \mathrm{~d} t\right)^{m}\left(\mathrm{e}^{y(0) / 2}\right)^{k}
$$

there was obtained its closed form. Nonetheless one can be easily convinced that such an approach to calculations is wrong, since the used series expansion (40) does not exist.

Thus, it is reasonable to mention some approximate methods of calculating the path integrals, in particular, for the case $\alpha=1$. Consider a formula for the option price (26). We will represent the corresponding path integrals in (26) as those with respect to the Gauss measure:

$$
C(t, S(t))=\int \mathrm{d} \mu(v)\left[S(t) F_{+}(v)-\mathrm{e}^{-\tau r} K F_{-}(v)\right]
$$

where $\mathrm{d} \mu(v)=\overline{\mathcal{N}}(\tau) \mathcal{D} v \mathrm{e}^{S_{\mathrm{G}}}$ - means a probabilistic Gauss integration measure. Here, the quantity $S_{\mathrm{G}}$ is given by the expression:

$$
S_{\mathrm{G}}=-\frac{1}{2 \xi^{2}} \int_{0}^{\tau}\left[v(t)+\mu-\xi^{2} / 2\right]^{2} \mathrm{~d} t
$$

and the next terms $F_{ \pm}(v)$ hold the remaining factors of formula (26). It is easy to see that the values $F_{ \pm}(v)$ are bound by $v \in \mathbb{R}$. Therefore, the integrals used in (41) exist. For an approximate calculation of path integrals (41) one can use numerical quadrature formulas, presented, in particular, in [18].

The use of approximation approaches is also of some interest. In solving the problems of quantum mechanics and statistical physics, the mean-path-approximation [1, 19] was effectively used. From this point of view let us rewrite formula (26) in the form:

$$
C(t, S(t))=\overline{\mathcal{N}}(\tau) \int \mathcal{D} v\left[S(t) \mathrm{e}^{S_{+}}-\mathrm{e}^{-\tau r} K \mathrm{e}^{S_{-}}\right]
$$

The mean-path approximations could be naturally considered as zero-approximations. In this case within the quantities $S_{ \pm}$we will make the change:

$$
\tilde{y}(t) \rightarrow y_{m}=\frac{1}{\tau} \int_{0}^{\tau} \tilde{y}(t) \mathrm{d} t=y-\frac{1}{\tau} \int_{0}^{\tau} t v(t) \mathrm{d} t .
$$


Upon the above mentioned change, the quantities $S_{ \pm}$will depend on the integration variables via the expressions:

$$
\int_{0}^{\tau} v(t) \mathrm{d} t, \quad \frac{1}{\tau} \int_{0}^{\tau} t v(t) \mathrm{d} t
$$

and the path integrals in (26) will then reduce to the usual quadratures. As a result, the price option formula will take the form:

$$
C(t, S(t))=\frac{2 \sqrt{3}}{\xi^{2} \tau} \mathrm{e}^{-\tau^{2}\left(\mu-\xi^{2} / 2\right) / 2} \int \frac{\mathrm{d} v_{1} \mathrm{~d} v_{2}}{2 \pi} \mathrm{e}^{S_{0}^{*}}\left[S(t) \mathrm{e}^{\tau \Delta r^{*}} N\left(d_{+}^{*}\right)-\mathrm{e}^{-\tau r} K N\left(d_{-}^{*}\right)\right],
$$

where the corresponding quantities are defined by the expressions:

$$
\begin{aligned}
S_{0}^{*}= & -\frac{1}{\xi^{2} \tau}\left(2 v_{1}^{2}+6 v_{2}^{2}-6 v_{1} v_{2}-\tau v_{1}\left[\mu-\xi^{2} / 2\right]\right)-\frac{\lambda \tau}{\xi^{2}} \mathrm{e}^{-y+v_{2}}\left(\mu-\xi^{2} / 2\right)- \\
& -\frac{\lambda^{2} \tau}{2 \xi^{2}} \mathrm{e}^{-2\left(y-v_{2}\right)}+\frac{\lambda}{\xi^{2}} \mathrm{e}^{-y}\left(1-\mathrm{e}^{v_{1}}\right)+\frac{\lambda \tau}{2} \mathrm{e}^{-y+v_{2}}, \\
d_{ \pm}^{*}= & \frac{\ln [S(t) / K]+\tau\left(r^{*} \pm \sigma^{* 2} / 2\right)}{\sigma^{*} \sqrt{\tau}}, \\
\sigma^{*}= & \left(1-\rho^{2}\right) \mathrm{e}^{y-v_{2}}, \\
r^{*}= & r-\frac{1}{2} \rho^{2} \mathrm{e}^{y-v_{2}}+\rho\left(\frac{\xi}{4}-\frac{\mu}{\xi}\right) \mathrm{e}^{\left(y-v_{2}\right) / 2}-\frac{2 \rho}{\xi} \mathrm{e}^{y / 2}\left(1-\mathrm{e}^{-v_{1}}\right), \\
\Delta r^{*}= & r^{*}-r .
\end{aligned}
$$

Our next step is to take the series corrections into account, if we expand the quantities $S_{ \pm}$into series with respect to suitable deviations $\Delta y(t)$ :

$$
\tilde{y}(t)=y_{m}+\Delta y(t)
$$

Certainly, the approaches mentioned above need a special investigation.

\section{Conclusions}

Using the Gaussian path integral, a representation of the evolution operator kernel for the Merton-Garman model was obtained. Also, some shortcomings that occurred in the cited sources [6, 7] were explained and corrected. A generalization of the well-known Black-Scholes formula for the rational price of European call option was also presented. Approximate approaches to the calculation of path integrals were also described.

Note also that the Black-Scholes formula (55) is widely used in practice in analyzing the options [9]. It is known that the analyzed model gives rise to quite good results in the case of well developed markets. However, shortcomings occur when a stock market is in an uncertainty state and the price dynamics swings [20]. At the same time, the Merton-Garman model should be considered as more realistic generalization of the Black-Scholes model taking into account the arbitrary volatility nature (formulas (7), (8) ). That is why the analysis of the obtained (26) and (39) formulas presents a considerable interest which will be the subject of our next investigations.

\section{Acknowledgments}

One of the authors (V. Yanishevsky) is especially grateful to A.K. Prykarpatsky for the fruitful discussions and the valuable comments and remarks. 


\section{A. The Black-Scholes model}

\section{A.1. The Black-Scholes equation}

The Black-Scholes equation [6, 14] describes the $C(t)$ option price dynamics:

$$
\frac{\partial C(t)}{\partial t}=H_{\mathrm{BS}} C(t),
$$

where $H_{\mathrm{BS}}$ denotes the following Black-Scholes Hamiltonian:

$$
H_{\mathrm{BS}}=-\frac{\sigma^{2}}{2} \frac{\partial^{2}}{\partial x^{2}}+\left(\frac{\sigma^{2}}{2}-r\right) \frac{\partial}{\partial x}+r .
$$

Equation (43) visually resembles a Schrodinger equation, written with respect to imaginary time, or a diffusion equation. Quantities $r$ and $\sigma$ are considered to be constant. The general solution of equation (43) is represented in the form:

$$
C(t)=\mathrm{e}^{t H_{\mathrm{BS}}} C(0),
$$

where $C(t)$ is the value in time $t, C(0)$ is an initial condition. When using equation (43) in order to evaluate the option prices it is convenient to study the dynamics backward in time. For a given value of $C(T)$ at the final moment of time $T$ we need to evaluate $C(t)$ at $t<T$. It follows from the solution of equation (45) that

$$
C(t)=\mathrm{e}^{-(T-t) H_{\mathrm{BS}}} C(T)=\mathrm{e}^{-\tau H_{\mathrm{BS}}} C(T),
$$

where we denoted $\tau=T-t$. Making use of the kernel $g\left(\tau, x-x_{0}\right)$, for expression (46) one obtains that:

$$
C(t, x)=\int_{-\infty}^{\infty} g\left(\tau, x-x_{0}\right) C\left(T, x_{0}\right) \mathrm{d} x_{0} .
$$

Based on (46) and (47) one finds the relationship:

$$
g\left(\tau, x-x_{0}\right)=\mathrm{e}^{-\tau H_{\mathrm{BS}}} \delta\left(x-x_{0}\right) .
$$

Since Hamiltonian (44) coefficients are constants, it is convenient to proceed to Fourier transform by the formula:

$$
f(t, x)=\frac{1}{\sqrt{2 \pi}} \int_{-\infty}^{\infty} \tilde{f}(t, k) \mathrm{e}^{\mathrm{i} k x} \mathrm{~d} k .
$$

In equation (43) it is convenient to proceed to Fourier transform, receiving the following result:

$$
\frac{\partial \tilde{C}(t, k)}{\partial t}=\tilde{H}_{\mathrm{BS}}(k) \tilde{C}(t, k),
$$

where $\tilde{C}(t, k)$ denotes Fourier transform of proper function and Fourier transform of Hamiltonian (44):

$$
\tilde{H}_{\mathrm{BS}}(k)=\frac{\sigma^{2}}{2} k^{2}+\mathrm{i}\left(\frac{\sigma^{2}}{2}-r\right) k+r .
$$

An equation (49) for a Fourier transform will be of the form:

$$
\tilde{C}(t, k)=\mathrm{e}^{-\tau \tilde{H}_{\mathrm{BS}}(k)} \tilde{C}(T, k) .
$$


Respectively for the kernel Fourier image $g\left(\tau, x-x_{0}\right)$ one has:

$$
\tilde{g}(\tau, k)=\mathrm{e}^{-\tau \tilde{H}_{\mathrm{BS}}(k)}
$$

Similarly, for the kernel of the evolution operator (49), applying inverse Fourier transform, we receive the following expression:

$$
\begin{aligned}
g\left(\tau, x-x_{0}\right) & =\frac{\mathrm{e}^{-\tau r}}{\sqrt{2 \pi}} \int_{-\infty}^{\infty} \tilde{g}(\tau, k) \mathrm{e}^{\mathrm{i} k\left(x-x_{0}\right)} \mathrm{d} k= \\
& =\frac{\mathrm{e}^{-\tau r}}{\sqrt{2 \pi}} \int_{-\infty}^{\infty} \exp \left(-\frac{1}{2} \tau \sigma^{2} k^{2}-\mathrm{i} \tau k\left[\sigma^{2} / 2-r\right]\right) \mathrm{e}^{\mathrm{i} k\left(x-x_{0}\right)} \mathrm{d} k= \\
& =\frac{\mathrm{e}^{-\tau r}}{\sqrt{2 \pi \tau \sigma^{2}}} \exp \left(-\frac{1}{2} \frac{\left(x-x_{0}+\tau\left[r-\sigma^{2} / 2\right]\right)^{2}}{\tau \sigma^{2}}\right) .
\end{aligned}
$$

\section{A.2. A Black-Scholes formula}

Let us recall a classical example of evaluation for the rational price of the European call option [6, 14]. A boundary condition (a payoff function) is defined in the form:

$$
C(T, S)=(S-K)^{+}= \begin{cases}S-K, & S>K \\ 0, & S \leqslant K\end{cases}
$$

where $K$ is strike price, $S$ is a market price at a moment $T$ (time to maturity). The payoff function (53) has a sufficiently obvious meaning - option transaction realizes if the stock market price is greater than the strike price. Option price at time $t<T$ is evaluated making use of the formula:

$$
\begin{aligned}
C(t, S(t)) & =\int_{-\infty}^{\infty} g\left(\ln [S(t)]-x_{0}\right)\left(\mathrm{e}^{x_{0}}-K\right)^{+} \mathrm{d} x_{0}= \\
& =\frac{\mathrm{e}^{-r \tau}}{\sqrt{2 \pi \sigma^{2}}} \int_{\ln (K)}^{\infty} \exp \left[-\frac{1}{2} \frac{\left(\ln [S(t)]-x_{0}+\tau\left[r-\sigma^{2} / 2\right]\right)^{2}}{\tau \sigma^{2}}\right]\left(\mathrm{e}^{x_{0}}-K\right) \mathrm{d} x_{0},
\end{aligned}
$$

where $S(t)$ denotes stock price at time $t$.

Having completed the necessary computations we receive, for option price [8], the well known Black-Scholes formula:

$$
C(t, S(t))=S(t) N\left(d_{+}\right)-K \mathrm{e}^{-r \tau} N\left(d_{-}\right)
$$

We have here denoted:

$$
N(x)=\frac{1}{2 \pi} \int_{-\infty}^{x} \mathrm{e}^{-z^{2} / 2} \mathrm{~d} z, \quad d_{ \pm}=\frac{\ln [S(t) / K]+\tau\left(r \pm \sigma^{2} / 2\right)}{\sigma \sqrt{\tau}} .
$$

\section{B. Gaussian path integrals}

The Gaussian path integral is used for the degree linearization in an $T$-exponential and looks as follows:

$$
\begin{aligned}
& T \exp \left(\int_{0}^{\tau} \frac{1}{2} A^{2}(t) \mathrm{d} t+\int_{0}^{\tau} B(t) d t\right)= \\
& =\mathcal{N}(\tau) \int \mathcal{D} v \exp \left(-\frac{1}{2} \int_{0}^{\tau} v^{2}(t) \mathrm{d} t\right) T \exp \left(-\int_{0}^{\tau} v(t) A(t) \mathrm{d} t+\int_{0}^{\tau} B(t) \mathrm{d} t\right) .
\end{aligned}
$$


In this work $A(t)$ and $B(t)$ - differential operators (in general not commutative), "the constant" $\mathcal{N}(\tau)$ is determined from the condition:

$$
\mathcal{N}(\tau) \int \mathcal{D} v \exp \left(-\frac{1}{2} \int_{0}^{\tau} v^{2}(t) \mathrm{d} t\right)=1, \quad \mathcal{D} v=\prod_{0 \leqslant t \leqslant \tau} \mathrm{d} v(t) .
$$

The Gaussian path integral (56) was used in [11] in constructing the path integral Green function representation for some quantum mechanical Hamiltonians.

It is evident that the field of application of the formula (56) is wide enough. The path integral (56) is interpreted as an operator identity. In particular, one can "disentangle" the factor $T \exp \left(\int_{0}^{\tau} B(t) \mathrm{d} t\right)$ in (56) in accordance with (63), and obtain the equivalent equality:

$$
T \exp \left(\frac{1}{2} \int_{0}^{\tau} \tilde{A}^{2}(t) \mathrm{d} t\right)=\mathcal{N}(\tau) \int \mathcal{D} v \exp \left(-\frac{1}{2} \int_{0}^{\tau} v^{2}(t) \mathrm{d} t\right) T \exp \left(-\int_{0}^{\tau} v(t) \tilde{A}(t) \mathrm{d} t\right),
$$

where

$$
\tilde{A}(t)=\left[T \exp \left(\int_{0}^{t} B\left(t^{\prime}\right) \mathrm{d} t^{\prime}\right)\right]^{-1} A(t) T \exp \left(\int_{0}^{t} B\left(t^{\prime}\right) \mathrm{d} t^{\prime}\right) .
$$

The integration in (57) is realized with respect to the Gaussian probability measure, since:

$$
\mathcal{N}(\tau) \int \mathcal{D} v \exp \left(-\frac{1}{2} \int_{0}^{\tau} v^{2}(t) \mathrm{d} t\right) T \exp \left(-\int_{0}^{\tau} v(t) \tilde{A}(t) \mathrm{d} t\right)=\int \mathrm{d} \mu(v) T \exp \left(-\int_{0}^{\tau} v(t) \tilde{A}(t) \mathrm{d} t\right)
$$

where $\mathrm{d} \mu(v)=\mathcal{N}(\tau) \mathcal{D} v \exp \left(-\frac{1}{2} \int_{0}^{\tau} v^{2}(t) \mathrm{d} t\right)$.

To check the identity (57), we expand the left and right hand sides in degree series. In particular, $T$-exponent on the left hand side (57) is represented as the degree series

$$
T \exp \left(\frac{1}{2} \int_{0}^{\tau} \tilde{A}^{2}(t) \mathrm{d} t\right)=\sum_{n=0}^{\infty} \frac{1}{n !} T\left(\frac{1}{2} \int_{0}^{\tau} \tilde{A}^{2}(t) \mathrm{d} t\right)^{n} .
$$

Expanding the right hand side (57) $T \exp \left(-\int_{0}^{\tau} v(t) \tilde{A}(t) \mathrm{d} t\right)$ in series and using the Gauss measure properties, one can derive expression (58). Really, for the left hand side of equality (57) one has:

$$
\int \mathrm{d} \mu(v) T \exp \left(-\int_{0}^{\tau} v(t) \tilde{A}(t) \mathrm{d} t\right)=\sum_{n=0}^{\infty} \int_{0}^{\tau} \mathrm{d} t_{1} \ldots \int_{0}^{\tau} \mathrm{d} t_{n} \frac{(-1)^{n}}{n !} \mu_{n} T\left[\tilde{A}\left(t_{1}\right) \ldots \tilde{A}\left(t_{n}\right)\right],
$$

where $\mu_{n}$ is the $n$-th Gauss measure correlation moment. As is well known, all uneven moments vanish, and the even ones are expressed by means of the second correlation moment:

$$
\mu_{2}=\int \mathrm{d} \mu(v) v\left(t_{1}\right) v\left(t_{2}\right)=\delta\left(t_{1}-t_{2}\right)
$$

The moment of $2 n$-th order is expressed by means of the moment $\mu_{2}$ as the relationship:

$$
\mu_{2 n}=\int \mathrm{d} \mu(v) v\left(t_{1}\right) \ldots v\left(t_{2 n}\right)=\sum_{P} \mu_{2}^{1} \ldots \mu_{2}^{n} .
$$


A summand in (60) is taken over all possible different smashes of $2 n$ elements by two-element subsets (see, for instance, [21]). The amount of summands in (60) equals

$$
\frac{(2 n) !}{2^{n} n !}
$$

Making use of symmetry properties (the permutations of operators under the $T$-product sign) it is not hard to demonstrate that all these summands give rise to the same result, thereby the formula (59) reduces to (58).

Based on (56) and the operator identity:

$$
2 A(t) B(t)=\frac{1}{2}(A(t)+B(t))^{2}-\frac{1}{2}(A(t)-B(t))^{2}+[A(t), B(t)]_{-},
$$

let us linearize the product of operators $A(t) B(t)$ :

$$
\begin{aligned}
& T \exp \left(\int_{0}^{\tau}[2 A(t) B(t)+D(t)] \mathrm{d} t\right)=\mathcal{N}(\tau)^{2} \int \mathcal{D} v \mathcal{D} u \exp \left(-\frac{1}{2} \int_{0}^{\tau} v^{2}(t) \mathrm{d} t-\frac{1}{2} \int_{0}^{\tau} u^{2}(t) \mathrm{d} t\right) \times \\
& \times T \exp \left(-\int_{0}^{\tau}\left\{[v(t)+\mathrm{i} u(t)] A(t)+[v(t)-\mathrm{i} u(t)] B(t)-[A(t), B(t)]_{-}-D(t)\right\} \mathrm{d} t\right) .
\end{aligned}
$$

Here we have used the following notation of the operator commutator $[A(t), B(t)]_{-}=A(t) B(t)-$ $B(t) A(t)$. Using the shift $v(t) \rightarrow v(t)+\mathrm{i} u(t)$ the right hand side of equation (61) can be written in the form:

$$
\begin{aligned}
& \mathcal{N}(\tau)^{2} \int \mathcal{D} v \mathcal{D} u \exp \left(-\frac{1}{2} \int_{0}^{\tau} v^{2}(t) \mathrm{d} t-\mathrm{i} \int_{0}^{\tau} v(t) u(t) \mathrm{d} t\right) \times \\
& \times T \exp \left(-\int_{0}^{\tau}\left\{[v(t)+2 \mathrm{i} u(t)] A(t)+v(t) B(t)-[A(t), B(t)]_{-}-D(t)\right\} \mathrm{d} t\right) .
\end{aligned}
$$

In the case of differential operators, the relationship (61) makes it possible to decrease its differential order. Note also that the path integral representation (61) is equivalent to the StratonovichHubbard identity [22] for the density matrix in quantum statistics problems.

Jointly with the introduced formulas, the following expression is used to extract $T$-exponent operators [13]:

$$
T \exp \left(\int_{0}^{\tau}[A(t)+B(t)] \mathrm{d} t\right)=T \exp \left(\int_{0}^{\tau} A(t) \mathrm{d} t\right) T \exp \left(\int_{0}^{\tau} \tilde{B}(t) \mathrm{d} t\right)
$$

where

$$
\tilde{B}(t)=\left[T \exp \left(\int_{0}^{t} A\left(t^{\prime}\right) \mathrm{d} t^{\prime}\right)\right]^{-1} B(t) T \exp \left(\int_{0}^{t} A\left(t^{\prime}\right) \mathrm{d} t^{\prime}\right) .
$$

\section{A connection between path integral approaches}

Consider the case for parameter values $\alpha=1, \lambda=0$. Based on (25) one can write down the kernel expression as:

$$
g\left(\tau, x-x_{0}, y, y_{0}\right)=\int_{y_{0}}^{y} \mathcal{D}^{\prime} y \mathrm{e}^{S_{0}} \frac{\mathrm{e}^{-\tau r}}{\sqrt{2 \pi \tau \tilde{\sigma}^{2}}} \exp \left(-\frac{1}{2} \frac{\left(x-x_{0}+\tau\left[\tilde{r}-\tilde{\sigma}^{2} / 2\right]\right)^{2}}{\tau \tilde{\sigma}^{2}}\right)
$$


The measure of integration in (64) satisfies the condition:

$$
\int_{y_{0}}^{y} \mathcal{D}^{\prime} y \exp \left[-\frac{1}{2 \xi^{2}} \int_{0}^{\tau} \dot{y}(t)^{2} \mathrm{~d} t\right]_{\tau \rightarrow 0} \rightarrow \delta\left(y-y_{0}\right)
$$

at the boundary values $y(0)=y_{0}, y(\tau)=y$. Moreover, in the above expressions (24) one should make the following substitutions:

$$
\tilde{y} \rightarrow y(t), \quad v(t) \rightarrow \dot{y}(t)
$$

Thereby, we obtain:

$$
\tilde{g}\left(\tau, x-x_{0}, y\right)=\int_{-\infty}^{\infty} \mathrm{d} y_{0} \int_{y_{0}}^{y} \mathcal{D}^{\prime} y \mathrm{e}^{S_{0}} \frac{\mathrm{e}^{-\tau r}}{\sqrt{2 \pi \tau \tilde{\sigma}^{2}}} \exp \left(-\frac{1}{2} \frac{\left(x-x_{0}+\tau\left[\tilde{r}-\tilde{\sigma}^{2} / 2\right]\right)^{2}}{\tau \tilde{\sigma}^{2}}\right)
$$

where

$$
\begin{aligned}
S_{0} & =-\frac{1}{2 \xi^{2}} \int_{0}^{\tau}\left[\dot{y}(t)+\mu-\xi^{2} / 2\right]^{2} \mathrm{~d} t \\
\tilde{\sigma}^{2} & =\left(1-\rho^{2}\right) \frac{1}{\tau} \int_{0}^{\tau} \mathrm{e}^{y(t)} \mathrm{d} t \\
\tilde{r} & =r-\frac{1}{2} \rho^{2} \frac{1}{\tau} \int_{0}^{\tau} \mathrm{e}^{y(t)} \mathrm{d} t+\rho\left(\frac{\xi}{4}-\frac{\mu}{\xi}\right) \frac{1}{\tau} \int_{0}^{\tau} \mathrm{e}^{y(t) / 2} \mathrm{~d} t-\frac{2 \rho}{\xi} \frac{1}{\tau}\left(\mathrm{e}^{y(\tau) / 2}-\mathrm{e}^{y(0) / 2}\right) .
\end{aligned}
$$

\section{References}

1. Feynman R.P., Hibbs A.R., Quantum Mechanics and Path Integrals. McGraw-Hill Book Company, New York, 1965.

2. Kleinert H., Path Integrals in Quantum Mechanics, Statistics, Polymer Physics and Financial Markets. 3-rd ed., World Scientific Publishing Co., Inc., River Edge, New Jersey, 2004.

3. Schulman L.S., Techniques and Applications of Path Integration. A Wiley-Interscience Publication, John Wiley \& Sons, Inc., New York, 1981.

4. Roepstorff G., Path Integral Approach to Quantum Physics. Springer, Berlin, 1994.

5. Wiegel F.W., Introduction to Path-Integral Methods in Physics and Polymer Science. World Scientific, Singapore, 1986.

6. Baaquie B.E., Quantum finance. Path Integrals and Hamiltonians for Options and Interest Rates, Cambridge University Press, New York, 2004; doi:10.1017/CBO9780511617577.008.

7. B.E. Baaquie, J. Phys. I, 1997, 7, No. 12, 1733; doi 10.1051/jp1:1997167.

8. Black F. and Scholes M., J. Polit. Econ., 1973, 81, No. 3, 637; doi $10.1086 / 260062$

9. Hull J.C., Options, Futures and Other Derivatives. 5-th ed., Prentice-Hall International, 2003.

10. Prohorov L.V., Physics of Elementary Particles and Atomic Nuclei (PEPAN), 1982, 13, No. 5, 1095 (in Russian).

11. Blazhijevsky L.F., Teor. Mat. Fiz., 1979, 40, No. 1, 51 (in Russian); [Engl. transl. Theor. Math. Phys., 1979, 40, No. 1, 596; doi 10.1007/BF01019242.

12. Grosche C., An Introduction into the Feynman Path Integrals. International School for Advanced Studies, Trieste, Miramare, Italy, 1992.

13. Nazajkinsky B.J., Sternin B.Y., Shalatov B.J., The Methods of Non-Commutive Analysis. Tehnosfera, Moscow, 2002 (in Russian).

14. Schyrajev A.N., The Fundamentals of Stohastic Financial Mathematics. V. 1,2, FAZIS, Moscow, 1998 (in Russian).

15. Balesku R., Equilibrium and Nonequilibrium Statistical Mechanics. Wiley-Interscience Publication, New York, 1975. 
16. Lindenberg K. and West B.J., The Nonequilibrium Statistical Mechanics of Open and Closed Systems. New York, 1990.

17. Hull J.C. and White A., J. Finance, 1987, 42 No. 2, 281; doi $10.2307 / 2328253$.

18. Jidkov J.P., Lobanov Y.Y., Physics of Elementary Particles and Atomic Nuclei (PEPAN), 1996, V. 27, No. 1, 173 (in Russian).

19. Feynman R.P., Statistical Mechanics. Benjamin, Reading, Massachusetts, 1972.

20. Nazarenko A.S., Ekonomical bulletin of the Rostov State University, 2007, 5, No. 1, 95 (in Russian).

21. Zinn-Justin J., Path Integrals in Quantum Mechanics, Oxford University Press, New York, 2005.

22. Masujima M., Path Integral Quantization and Stochastic Quantization. Springer-Verlag, Berlin, 2009.

\title{
Континуальне представлення ядра оператора еволюції в моделі Мертона-Кармана
}

\author{
Л.Ф. Блажиєвський \\ 1 Кафедра теоретичної фізики, Львівський Національний університет імені Івана Франка, \\ вул. Драгоманова, 12, 79005 Львів, Україна \\ 2 Кафедра економічної кібернетики та інноватики, Дрогобицький державний педагогічний \\ університет імені Івана Франка, вул. Лесі Українки, 82100 Дрогобич, Україна
}

В методі континуального інтегрування побудовано ядро оператора еволюції для гамільтоніану Мертона-Кармана. На основі ядра отримана формула для ціни опціону, що узагальнює відому формулу Блека-Шоулса. Вказано також на можливі способи наближеного обчислення континуальних інтегралів.

Ключові слова: континуальне інтегрування, ядро оператора еволюції, ціноутворення опціонів, формула Блека-Шоулса, модель Мертона-Кармана. 